\title{
MORFOLOGIA FLORAL DA GOIABEIRA SERRANA (Feijoa sellowiana) E SUAS IMPLICAÇÕES NA POLINIZAÇÃ̃O ${ }^{1}$
}

\author{
JULIANA DEGENHARDT ${ }^{2}$, AFONSO INÁCIO ORTH ${ }^{3}$, MIGUEL PEDRO GUERRA ${ }^{3}$, \\ JEAN-PIERRE DUCROQUET ${ }^{4}$, RUBENS ONOFRE NODARI ${ }^{3}$
}

\begin{abstract}
RESUMO - Esse estudo teve por objetivo caracterizar variações na arquitetura floral da Feijoa sellowiana e determinar sua influência sobre a polinização. Foram avaliados o comprimento do pistilo, a distância entre estigma e estames, o diâmetro de abertura e a distribuição dos estames na flor, com base em 7 flores de 15 acessos do Banco de Germoplasma da espécie. Distinguiram-se 3 classes de distâncias médias entre estigma e estames: $0,2(\mathrm{C} 1), 0,7(\mathrm{C} 2)$ e 1,2 cm (C3), e duas classes de distribuição de estames na flor (radial e aleatória). Sorteou-se uma planta por classe de distância entre estigma e estames, a qual teve 50 flores marcadas para cada um dos seguintes tratamentos: T1-polinização aberta; T2-tela contra pássaros e T3-tela contra pássaros e insetos. A frutificação foi de $47 \% \mathrm{em}$ T1 para C2 e de $22 \%$ para C3, diferença que pode ser devida ao genótipo e/ou alternância de produção. No T2, a frutificação foi $31 \%$ em $\mathrm{C} 2,15,4 \% \mathrm{em} \mathrm{C} 1$ e $3 \%$ em C3. A superioridade de $\mathrm{T} 1$ sobre $\mathrm{T} 2$ pode ser devida à polinização por pássaros. Essa superioridade foi mais expressiva em $\mathrm{C} 3$ do que em $\mathrm{C} 2$, o que sugere que $\mathrm{C} 3$ é mais dependente de pássaros. A frutificação em T2 pode ainda evidenciar a polinização por insetos e foi menor para $\mathrm{C} 3$, o que pode estar sendo causado pelo maior afastamento do estigma. A frutificação em $\mathrm{T} 3$ para C1 (7\%) e C2 (15\%) sugere que o vento ou a autopolinização também possam efetuar a polinização.
\end{abstract}

Termos para indexação: Myrtaceae, Acca sellowiana, arquitetura floral.

\section{FLOWER MORPHOLOGY OF FEIJOA (Feijoa sellowiana) AND IT'S IMPLICATIONS ON POLLINATION}

\begin{abstract}
S - The objective of our research was to evaluate distance between the stigma and the stamens, opening diameter and stamens distribution on the flower. We evaluated these variables on 7 flowers of each one of the 15 plant accessions from the Germplasm Bank. The flowers were classified according to distance between the stigma and the stamens into three classes: 0.2 (C1), 0.7 $(\mathrm{C} 2)$ and 1,2 (C3) cm, in which 2, 9, and 4 accessions were included, respectively. The radial and random stamens distributions were verified in 6 and 9 accessions, respectively. These two variables behaved independently. Thereafter one plant were randomly taken from each class and marked 150 flowers, being 50 for each of the following treatments: T1 - natural pollination; T2 - screen which excluded only the birds from reaching the flowers; and T3 - screen which excluded both birds and insects. It was evaluated the fruit set in all three treatments. We verified $47 \%$ of fruit set in $\mathrm{T} 1$ of $\mathrm{C} 2$ and $22 \%$ in $\mathrm{T} 1$ of $\mathrm{C} 3$. This difference can be due to genotypic differences and/or alternation of yield. In T2, fruit set was $31 \%$ in $\mathrm{C} 2,15.4 \%$ in $\mathrm{C} 1$, and $3 \%$ in $\mathrm{C} 3$. The superiority in fruit set of T1 over T2 can be credited to bird pollination. The fruit set in $\mathrm{T} 1$ was 7 times larger in $\mathrm{C} 3$ and 2 times larger in $\mathrm{C} 2$, than in $\mathrm{T} 2$. This suggests that $\mathrm{C} 3$ is more dependent on birds for pollination. The observed fruit set in $\mathrm{T} 2$ can be partially credited to insect pollination and was smaller in $\mathrm{C} 3$, as expected due to the larger pistils. Even in T3 we observed fruit set in C1 (7\%) and in C2 (15\%), which suggests that either wind plays a role in the pollination or that selfing may occur. Since there was no fruit set in T3 on C3, presence of autoincompatibility cannot be discarded.
\end{abstract}

Index terms: Myrtaceae, Acca sellowiana, pineapple-guava, floral morphology.

A Feijoa sellowiana é uma mirtácea nativa do planalto meridional brasileiro, que vem sendo pesquisada devido ao potencial de seus frutos. A planta é um arbusto ou pequena árvore que entra em produção a partir do quarto ano. Suas flores são desprovidas de nectários e apresentam quatro pétalas vistosas, carnosas e adocicadas, que são o principal recurso floral para os polinizadores (Mattos, 1986). A flor apresenta em média 60 estames e um estigma situado normalmente acerca de 5 a $7 \mathrm{~mm}$ acima do plano das anteras (Ducroquet et al., 2000).

A flor é bispórica e longistilada, com tendência à dicogamia por protoginia, pelo fato de o estigma tornar-se receptivo 24 horas antes da deiscência das anteras, porém permanecendo receptivo ainda por 10 horas após a deiscência (Stewart \& Craig, 1989). Entretanto, avaliações em acessos do Banco de

1 (Trabalho 134/2000). Recebido: 12/07/2000. Aceito para publicação: 18/10/2001.

2 Mestre em Recursos Genéticos Vegetais, UFSC/CCA, Depto de Fitotecnia, CP 476, CEP 88040-900, Florianópolis, SC; julianadt@hotmail.com 3 Prof Doutor do CPG em Recursos Genéticos Vegetais, CCA/UFSC

4 Pesquisador Doutor da EPAGRI - Videira/SC 
Germoplasma da espécie, em Videira-SC, mostraram que alguns destes são autocompatíveis (Ducroquet et al., 2000).

Devido à arquitetura floral, Popenoe (1912) sugeriu que a polinização fosse realizada principalmente por meio de pássaros que visitam as flores com o interesse de comer suas pétalas e acabam por transferir o pólen ao roçar seu peito contra as flores. Os insetos teriam essa função dificultada, devido a distância entre o estigma e o plano das anteras (Ducroquet \& Hickel, 1997). Segundo Stewart \& Craig (1989), embora tanto pássaros quanto insetos tenham visitado as flores da goiabeira serrana, apenas duas espécies de pássaros foram polinizadores efetivos na Nova Zelândia (Turdus mercula e Acridotheres tristis).

No Brasil, a polinização é garantida por várias espécies de pássaros frugívoros (sanhaços, sabiás, saíras, gaturamos e tuques) (Zimmermann \& Orth, 1999; Hickel \& Ducroquet, 2000). Contudo, para as condições catarinenses, além dos pássaros, insetos himenópteros da superfamília Apoidea também participam da polinização. Estudos recentes mostraram que as mamangavas de toco, Xylocopa augusti e X. frontalis, e as de chão, Bombus atratus, tocam o estigma e as anteras durante as visitas florais (Hickel \& Ducroquet, 2000). A Apis mellifera é ineficiente na polinização de plantas autoincompatíveis, mas pode polinizar as autocompatíveis (Hickel \& Ducroquet, 2000).

Este estudo teve por objetivos caracterizar a variação na distância entre estigma e estames em flores de plantas de goiabeira serrana; caracterizar a variação da distribuição dos estames na flor e avaliar a contribuição dos diferentes agentes polinizadores dentre as diferentes classes de arquitetura floral previamente determinadas, para avaliar a influência de variações na arquitetura floral sobre a polinização da espécie.

As avaliações foram realizadas de novembro de 1999 a janeiro de 2000 no Banco de Germoplasma de São Joaquim (SC). Inicialmente, foi medida a distância entre o estigma e os estames e o comprimento total dos pistilos de 7 flores de 15 acessos, quando as mesmas encontravam em estádio fenológico F2 (conforme Ducroquet \& Hickel, 1991). Foram então estabelecidas três classes, de acordo com essa distância (Figura 1): C 1- Flores com distância variando entre 0 e $0,4 \mathrm{~cm} ; \mathrm{C} 2$ - Flores com distância variando entre 0,5 e $0,9 \mathrm{~cm} ; \mathrm{C} 3$ - Flores com distância variando entre 1,0 e $1,4 \mathrm{~cm}$.

Em seguida, foram avaliados a distribuição espacial dos estames e o diâmetro de abertura do conjunto. Os acessos foram distribuídos em duas classes (Figura 1): Classe A - Flores com estames distribuídos de maneira aleatória, com alguns destes localizados próximo ao pistilo; Classe B - Flores com estames distribuídos de forma radial, localizando-se a maiores distâncias do pistilo.

Para verificar as variações durante os diferentes estágios fenológicos da flor, foram realizadas medições em três flores marcadas em um acesso de cada classe de distância entre estigma e estames: 1 (acesso 300), 2 (acesso 509) e 3 (acesso 141), durante o período de três dias, desde o estágio de recém-aberta, até o estágio em que as pétalas se encontravam completamente voltadas para baixo, fase em que o estigma não estava mais receptivo.

Foi então escolhida uma planta dentro de cada classe de distância entre estigma e estames (C1- acesso 374, autoincompatível; C2 - acesso 132 e C3 - acesso 141), nas quais foram marcadas 50 flores para cada um dos tratamentos a seguir:
$\mathrm{T} 1$ - Controle - Flores polinizadas naturalmente; $\mathrm{T} 2$-Flores com tela contra pássaros; T3 - Flores com tela contra pássaros e insetos.

Foram feitas as seguintes análises estatísticas: média e desvio-padrão das características avaliadas em 7 flores de cada acesso; teste de $\mathrm{c}^{2}$ para verificar se a proporção de acessos foi: (a) 1:1:1 nas três classes de distância; (b) 1:1 nas classes de distribuição de estames; e (c) se há independência entre estas duas variáveis; t-teste para verificar diferenças entre médias de comprimento do pistilo e diâmetro de abertura dos estames para as três classes de distância e correlações de Pearson entre as variáveis avaliadas (Beiguelman, 1991).

O comprimento médio do pistilo variou de 2,5 a $3,4 \mathrm{~cm}$. Os valores de desvio-padrão das médias (de 0,08 a $0,32 \mathrm{~cm}$ ) foram relativamente baixos, indicando que o tamanho do pistilo é praticamente o mesmo em todas as flores de uma mesma planta (Tabela 1). Também, o diâmetro médio de abertura dos estames, que variou de 2,6 a 4,4 cm entre os acessos, é típico de cada árvore, uma vez que o desvio-padrão oriundo da medida de 7 flores de cada acesso foi baixo $(0,13 \mathrm{a} 0,48 \mathrm{~cm})$.

Apenas 13,3\% das plantas apresentaram flores com distância média entre estigma e estames inferior a $0,5 \mathrm{~cm}$ (Tabela 2). A maioria dos acessos $(60,0 \%)$ apresentou distância média intermediária e $26,7 \%$ apresentaram distância média igual ou superior a $1,0 \mathrm{~cm}$. A soma dos desvios da proporção de 1:1:1 não atingiu valores estatisticamente significativos $\left(\mathrm{c}^{2}=5,20\right.$; $0,05<\mathrm{P}<0,10$ ). Contudo, esta hipótese necessita ser validada em um número maior de amostras.

Quanto à classificação baseada na distribuição dos estames na flor, houve uma distribuição bastante uniforme entre as duas classes (Tabela 2, Figura 1), sendo que seis acessos apresentaram distribuição radial e nove acessos apresentaram distribuição aleatória $\left(\mathrm{c}^{2}(=0,62 ; 0,30<\mathrm{P}<0,50)\right.$. Não foi detectada relação de dependência entre as duas classificações $\left(c^{2}=2,26\right.$; $0,30<\mathrm{P}<0,50$ ).

O comprimento médio do pistilo apresentou variação entre as classes de distância entre estigma e estames. A partir do teste t, verificaram-se diferenças estatísticas $(\mathrm{P}<0,01)$ entre as médias das classes 1 e $3(\mathrm{t}=4,66)$ e as médias das classes 2 e $3(\mathrm{t}=3,63)$. Esse resultado sugere que a distância entre o estigma e os estames seja influenciada por diferenças no comprimento do pistilo, porém diferenças no comprimento dos estames também devem existir, uma vez que não houve diferença significativa entre o comprimento médio de pistilo das classes 1 e 2 .

O diâmetro de abertura do conjunto de estames não apresentou grande variação entre as classes de distância entre estigma e estames ( $3,4 \mathrm{~cm}$ para as flores da $\mathrm{C} 2$ e C 3 e 3,3 cm para $\mathrm{C} 1)$. Isso sugere que não há diferenças expressivas quanto ao diâmetro de abertura dos estames para as diferentes classes. Pelo teste $t$, não se constatou diferença significativa entre os valores desta medida entre as classes $(\mathrm{P}<0,01)$.

A correlação obtida foi expressiva entre comprimento de pistilo e distância entre estigma e estames $(0,82 ; \mathrm{P}<0,01)$ e de baixa magnitude entre comprimento de pistilo e diâmetro de abertura dos estames $(0,37 ; \mathrm{P}<0,01)$. O coeficiente de correlação entre distância do estigma e estames e diâmetro de abertura dos estames não foi significativo.

A distância entre estigma e estames pode ser um fator importante na seleção de polinizadoares. Tecnologias que 
TABELA 1- Média e desvio-padrão (DP) do comprimento de pistilo, distância entre estigma e estames e diâmetro de abertura dos estames avaliados em 7 flores de 15 acessos do Banco de Germoplasma de goiabeira serrana. São Joaquim-SC, 2000.

\begin{tabular}{|c|c|c|c|c|c|c|}
\hline A cesso & \multicolumn{2}{|c|}{ Comprim entodo pistilo } & \multicolumn{2}{|c|}{ 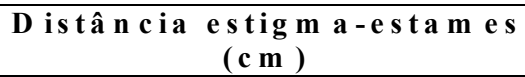 } & \multicolumn{2}{|c|}{$\begin{array}{c}\text { Diâmetrode abertura dos } \\
\text { estames (cm) }\end{array}$} \\
\hline 509 & 2,9 & 0,26 & 0,6 & 0,12 & 3,1 & 0,18 \\
\hline 141 & 3,4 & 0,25 & 1,4 & 0,14 & 2,7 & 0,38 \\
\hline $85 \mathrm{C}$ & 3,2 & 0,08 & 1,0 & 0,11 & 3,8 & 0,25 \\
\hline 300 & 2,5 & 0,09 & 0,2 & 0,05 & 3,4 & 0,32 \\
\hline 504 & 2,7 & 0,18 & 0,5 & 0,11 & 3,3 & 0,29 \\
\hline 141 & 3,4 & 0,09 & 1,2 & 0,13 & 3,0 & 0,29 \\
\hline 101 & 3,1 & 0,14 & 0,6 & 0,07 & 4,4 & 0,40 \\
\hline 370 & 3,4 & 0,27 & 1,0 & 0,18 & 4,2 & 0,38 \\
\hline 512 & 2,9 & 0,21 & 0,5 & 0,20 & 3,1 & 0,20 \\
\hline 374 & 2,8 & 0,16 & 0,2 & 0,07 & 3,5 & 0,38 \\
\hline 376 & 3,4 & 0,31 & 0,7 & 0,05 & 3,8 & 0,39 \\
\hline
\end{tabular}

TABELA 2 - Diâmetro de abertura de estames, comprimento médio do pistilo e número de acessos de acordo com as classes de distância entre o estigma e estames e classes de distribuição dos estames na flor. São Joaquim-SC, 2000.

\begin{tabular}{|c|c|c|c|c|c|c|}
\hline \multirow[b]{3}{*}{ C lasses } & \multirow{3}{*}{$\begin{array}{c}\text { Intervalo de } \\
\text { C lasse } \\
(\mathrm{c} \mathrm{m})\end{array}$} & \multirow{3}{*}{$\begin{array}{l}\text { D iâm etrode } \\
\text { abertura dos } \\
\text { estam es } \\
(\mathrm{cm})\end{array}$} & \multirow{3}{*}{$\begin{array}{c}\text { Comprimento } \\
\text { m édio do } \\
\text { pistilo (cm })\end{array}$} & \multicolumn{3}{|c|}{$\mathrm{N}$ úm ero de acessos } \\
\hline & & & & \multirow{2}{*}{$\begin{array}{c}\text { Por classe } \\
\text { de distância }\end{array}$} & \multicolumn{2}{|c|}{$\begin{array}{c}\text { Por distribuição de } \\
\text { estames }\end{array}$} \\
\hline & & & & & A leatória & $\mathrm{R}$ a dial \\
\hline C 2 & $0,5-0,9$ & 3,4 & 2,9 & 9 & 5 & 4 \\
\hline C 3 & $1,0-1,4$ & 3,4 & 3,3 & 4 & 2 & 2 \\
\hline
\end{tabular}

TABELA 3- Evolução do valor da distância entre estigma e estames e diâmetro de abertura dos estames. São Joaquim-SC, 2000.

\begin{tabular}{|c|c|c|c|c|c|c|}
\hline \multirow[b]{2}{*}{ A c e s s o } & \multicolumn{3}{|c|}{ D istân cia (cm) } & \multicolumn{3}{|c|}{ D iâmetro (c m) } \\
\hline & $1^{\circ} \mathrm{d}$ i a & $2^{\circ} \mathrm{d}$ ia & $3^{\circ}$ d i a & $1^{\circ} \mathrm{d}$ ia & $2^{\circ} \mathrm{d}$ ia & $3^{\circ} \mathrm{d}$ i a \\
\hline 300 & 0,2 & 0,3 & 0,3 & 2,0 & 3,0 & 4,4 \\
\hline 300 & 0,2 & 0,3 & 0,3 & 2,4 & 3,2 & 3,8 \\
\hline 300 & 0,2 & 0,4 & 0,4 & 2,0 & 3,4 & 4,0 \\
\hline 509 & 0 & 0,9 & 0,9 & 1,2 & 4,0 & 4,0 \\
\hline 509 & 0,5 & 0,6 & 0,8 & 2,0 & 3,0 & 3,4 \\
\hline 509 & 0,4 & 0,6 & 0,8 & 3,0 & 3,0 & 4,0 \\
\hline 141 & 0,5 & 1,4 & 1,6 & 2,0 & 3,0 & 3,1 \\
\hline 141 & 0,2 & 1,2 & 1,2 & 2,0 & 2,8 & 3,5 \\
\hline 141 & 0,2 & 1,3 & 1,3 & 2,5 & 3,4 & 4,4 \\
\hline
\end{tabular}

envolvam a utilização de agentes polinizadores com tamanho corporal menor como, por exemplo, Apis mellifera, seriam mais adequadas aos acessos que apresentam menor distância. Na cultura do maracujá, insetos menores do que as mamangavas dificilmente polinizam as flores devido a grande distância existente entre o nectário e o estigma (Manica, 1981). Free (1993) apontou abelhas grandes como algumas espécies de Xylocopa como principais polinizadores desta cultura.

No experimento onde as flores foram acompanhadas no tempo, verificou-se que as mesmas já apresentavam diferenças no comprimento do pistilo desde recém-abertas. Pôde-se observar também que, para as flores de todas as classes, no primeiro dia, o estigma se encontrava ainda enrolado, localizando-se por isso no mesmo nível das anteras. Já no segundo dia, as pétalas encontravam-se em ângulo de $90^{\circ}$, e os estigmas já se encontravam a uma distância dos estames de acordo com a classe à qual as plantas pertenciam. Esse é o estádio onde ocorre a deiscência das anteras e, portanto, é o estádio onde os insetos visitam as flores, à procura de pólen. Como nesse período o estigma se encontra receptivo, essa é a fase mais importante no tocante à polinização entomófila. No terceiro dia, as pétalas já se encontravam em ângulo superior a $90^{\circ}$ e o diâmetro de abertura dos estames foi maior para todas as classes (Tabela 2).

Os resultados da avaliação da porcentagem de frutificação estão apresentados na Figura 2. As diferenças na frutificação, no tratamento sem tela (T1), provavelmente estejam sendo causadas por diferenças nos genótipos das plantas, alternância de produção ou autocompatibilidade. Também é possível que as plantas da classe 3 apresentem menor porcentagem de frutificação pelo fato de serem mais dependentes de pássaros, devido a maior distância entre o estigma e os estames. Os dados da classe 1 não foram obtidos para este tratamento.

Nas flores protegidas com telas para pássaros (T2), a frutificação foi maior na classe 2, intermediária na classe 1 e menor na classe 3. A maior frutificação no T1 em relação ao T2 pode ser devida à polinização por pássaros. A frutificação foi 7 vezes 


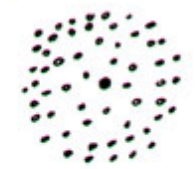

Classe A

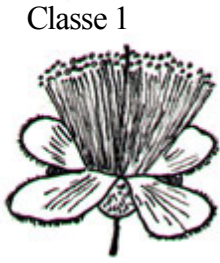

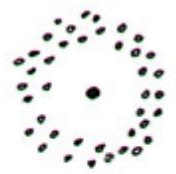

Classe B
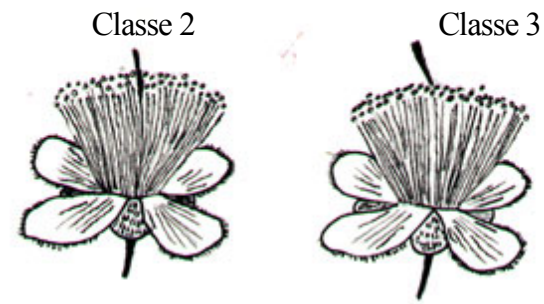

FIGURA 1 - Padrões de distribuição de estames e distância entre estigma e estames em Feijoa sellowiana. Classe A - Distribuição de estames aleatória; Classe B Distribuição de estames radial. Classes 1, 2 e 3 distância entre o estigma e os estames variando entre 0 e $0,4 \mathrm{~cm} ; 0,5$ e $0,9 \mathrm{~cm}$ e 1,0 e $1,4 \mathrm{~cm}$, respectivamente. São Joaquim-SC, 2000.

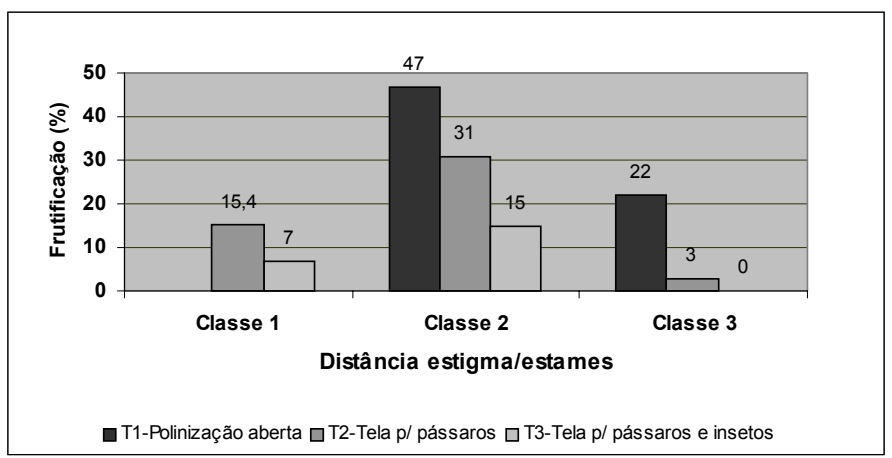

FIGURA 2 - Porcentagem de frutificação efetiva. Classes 1, 2 e 3 - distância entre o estigma e os estames variando entre 0 e $0,4 \mathrm{~cm} ; 0,5$ e $0,9 \mathrm{~cm}$ e 1,0 e $1,4 \mathrm{~cm}$, respectivamente. São Joaquim-SC, 2000.

maior na classe 3 e 2 vezes na classe 2 , o que sugere que a polinização na classe 3 é mais dependente de pássaros. $O$ fato de ocorrer frutificação no T2 pode ainda evidenciar a polinização realizada por insetos, e foi menor na classe 3 , como esperado, devido a maior distância entre o estigma e as anteras.

Mesmo no tratamento 3 (com tela para insetos e pássaros), houve frutificação para as classe 1 e 2 , o que sugere a presença de polinização anemófila, pois, no primeiro caso, o genótipo é autoincompatível, ou que a autopolinização possa ocorrer no acesso da classe 2. Como não houve frutificação na classe 3 , a existência de autoincompatibilidade não pode ser descartada e já vem sendo demonstrada em alguns acessos do Banco.

Os experimentos deverão ser repetidos, abrangendo a análise de um maior número de fatores que podem estar interferindo na polinização. É importante, ainda, que sejam realizados cruzamentos entre os acessos das diferentes classes, para que se possam estudar as bases genéticas das características determinantes da arquitetura floral, a fim de utilizá-las em programas de melhoramento da espécie.

Conclui-se então que:

1. Existe variação para as características distância entre o estigma e as anteras e distribuição dos estames ao redor do estilete entre os acessos, e esta não é devida ao estágio de desenvolvimento da flor.

2. As variáveis distância entre estigma e estames e distribuição dos estames se comportam de maneira independente.

3. Os resultados mostraram ainda a existência de uma associação entre classes morfológicas e a porcentagem de frutificação observada.

\section{REFERÊNCIAS BIBLIOGRÁFICAS}

BEIGUELMAN, B. Curso prático de bioestatística. 2. ed. Ribeirão Preto: SBG, 1991.231p.

DUCROQUET J.P.H.J.; HICKEL, E.R. Fenologia da goiabeira serrana (Feijoa sellowiana Berg) no alto vale do Rio do Peixe, Santa Catarina. Revista Brasileira de Fruticultura, Cruz das Almas, v.13, n.3, p.313-320, 1991.

DUCROQUET, J.P.H.J; HICKEL, E., R. Birds as pollinators of Feijoa (Acca sellowiana Berg). Acta Horticulturae, Leuven, n.452, p.3740, 1997.

DUCROQUET, J.P.H.J., HICKEL, E., R., NODARI, R. O. Goiabeira serrana (Feijoa sellowiana). Jaboticabal: FUNEP, 2000. 66p. (Série Frutas Nativas, 5)

FREE, J.B. Insect pollination of crops. 2. ed. London: Academic Press, 1993.684p.

HICKEL, E., R.; DUCROQUET, J.P.H.J. Polinização entomófila da goiabeira serrana, Feijoa selowiana (Berg.) em Santa Catarina. Revista Brasileira de Fruticultura, Jaboticabal, v.22, n.1, p.96101,2000 .

MANICA, I. Fruticultura tropical: maracujá. São Paulo: Agronômica Ceres, 1981, 160p.

MATTOS, J.R. A goiabeira serrana Porto Alegre: Instituto de Pesquisas de Recursos Naturais Renováveis, 1986. 84p. (Publicação IPRNR, 19)

POPENOE, F.W. Feijoa sellowiana, its history, culture and varieties. Pomona College Journal of Economic Botany, v.2, n.1, p.217-242, 1912.

STEWART, A. M.; CRAIG, J. L. Factors affecting pollinator effectiveness in Feijoa sellowiana. New Zealand Journal of Crop and Horticultural Science, Auckland, v.17, p.145-154, 1989.

ZIMMERMANN, C. E.; ORTH, A. I. Avifauna explorando as flores da goiabeira serrana Acca sellowiana (Berg) Burret em São Joaquim, Santa Catarina; In: CONGRESSO NACIONAL DE BOTÂNICA, 50., 1999, Blumenau, SC. Anais... Blumenau: SBB, 1999. p.218. 